\title{
ENTRE EL PETRÓLEO Y LA DEMOCRACIA: EL MECANISMO CAUSAL QUE UNE LA LIBERALIZACIÓN PETROLERA Y EL FORTALECIMIENTO DE CONTROLES DEMOCRÁTICOS EN LA POLÍTICA PETROLERA COLOMBIANA (2002-20I 8)*
}

IVÁN Bolívar RuIZ-GonZÁLEZ ${ }^{* *}$

\section{Resumen}

Este artículo explora la relación causal entre liberalización petrolera y controles democráticos. Parte de un análisis neoinstitucional de la política petrolera conjuntamente con el marco analítico del diseño de políticas desde los instrumentos NATO (nodalidad, autoridad, tesoro y organización). Con el fin de comprobar la relación hipotética, la investigación concibe la causalidad de una manera realista, utilizando el rastreo de procesos como método para descubrir el mecanismo causal intermedio, junto a una epistemología bayesiana para formalizar los resultados de la investigación. Al final se concluye que dentro de la relación causal existe un mecanismo intermedio, con fuerte presencia del mercado; además, expone el posicionamiento de nuevos sujetos políticos determinantes para mejorar la rendición de cuentas públicas.

Palabras clave: petróleo, controles democráticos, mecanismo causal, rastreo de procesos, liberalización.

\footnotetext{
* El autor agradece las observaciones y sugerencias de dos revisores o revisoras anónimos para mejorar la calidad del presente artículo. Como es usual, el autor asume la responsabilidad por el contenido o las omisiones en esta investigación. ${ }^{*}$ Maestro en Políticas Públicas por Flacso Ecuador. Asistente de investigación dentro del proyecto de investigación de políticas públicas comparadas, Flacso-Ecuador (Ecuador).

[ivanruiz-pm@hotmail.com], [https://orcid.org/0000-0002-3837-4708].

Recibido: 18 de noviembre de 2019 / Modificado: 28 de enero de 2020 / Aceptado: 13 de febrero de 2020

Para citar este artículo

Ruiz-González, I. B. (2020). Entre el petróleo y la democracia: el mecanismo causal que une la liberalización petrolera y el fortalecimiento de controles democráticos en la política petrolera colombiana (2002-2018). OPERA, 27, 205-224 DOI: https://doi.org/10.18601/16578651.n27.10
} 
BETWEEN OIL AND DEMOCRACY:THE CAUSAL MECHANISM THAT LINKS OIL LIBERALIZATION AND THE STRENGTHENING OF ACCOUNTABILITY IN COLOMBIAN OIL POLICY (2002-2018)

\section{Abstract}

This article explores the causal relationship between oil liberalization and accountability. It starts from a neo-institutional analysis of public policies after analyzing the oil policy according to NATO instruments under the analytical framework of policy design. In order to detect the hypothetical relationship, the investigation conceives causality in a realistic way, using process tracing as a method to discover the intermediate causal mechanism, together with a Bayesian epistemology that formalizes the research results. In the end it concludes that within the causal relationship there is an intermediate mechanism with a strong market presence. In addition, it exposes the positioning of new determining political subjects to improve public accountability.

Key words: Oil; accountability; causal mechanism; process tracing; liberalization.

\section{INTRODUCCIÓN: LIBERALIZACIÓN PETROLERA Y CONTROLES DEMOCRÁTICOS}

Latinoamérica presenta un contexto de debilidad institucional caracterizada por la desigualdad socioeconómica, la permanencia de una débil capacidad estatal y una alta volatilidad económica y política (Brinks, Levitsky y Murillo, 2019, pp. 50-52). En este contexto, varios países latinoamericanos optaron por la liberalización de sus economías desde la década de los noventa. Esta tendencia se redujo con la efervescencia de gobiernos de izquierda, sin embargo, aún subsisten Estados que apuestan por el mercado como agente de desarrollo.

En su acepción clásica, el liberalismo propone reducir la intervención estatal en la economía, dejando la regulación a las fuerzas del mercado que logran, por sí mismas, mejorar la eficiencia económica y promover el crecimiento (Smith, 1958; Ganuza, Paes de Barros y Vos, 2001, p. 79). La eficiencia se basa en la competencia, donde los ofertantes están obligados a mejorar la calidad de sus productos o servicios para ser más competitivos. Por esta razón, la participación de capital privado entra en juego, pues surge la necesidad de invertir o innovar para aumentar los ingresos y la competitividad.

La liberalización permeó en la política petrolera al impulsar reformas en las empresas nacionales de petróleo (State Oil Company, en adelante soc), y reducciones del control estatal en esta área (Balza y Espinasa, 2015). Fue implementada de acuerdo con un estilo de gobierno acorde con los supuestos ideológicos del liberalismo económico. Es decir, la reducción de la intervención del Estado y la búsqueda de eficiencia mantienen una alineación política con un modo de gobierno similar al de la administración privada (managerialismo). Al contemplar un control estatal reducido, la liberalización asume un tipo específico de descentramiento del Estado, desplazándolo hacia afuera (Pierre y Peters, 2000, p. 77).

Actualmente, Latinoamérica afronta un problema crónico de corrupción, ligado ínti- 
mamente a la debilidad de sus instituciones; en este escenario, el (neo)liberalismo ${ }^{1}$ se presentó como una alternativa plausible de transformación del Estado. Opera como un paradigma ${ }^{2}$, con instituciones propias, para orientar el comportamiento político hacia lo que se considera correcto bajo su paraguas. La necesidad de hacer económicamente eficiente al sector petrolero obliga a los operadores políticos y económicos a actuar bajo reglas prescriptivas de acción sobre lo adecuado para lograr el fin propuesto. La política petrolera, orientada bajo las normas de liberalización, crea reglas y rutinas que perfilan el comportamiento de los actores. Los predispone a buscar eficiencia y eficacia en la administración del sector petrolero mediante el aperturismo ${ }^{3}$.

Algunos países con régimen aperturista asumieron la liberalización como objetivo de

1 Dentro del artículo se entiende por neoliberalismo como el retorno hacia las leyes de mercado en reemplazo del Estado, implantar un sistema en el que el Estado se inhiba de intervenir en la economía, abrir las fronteras a la inversión extranjera indiscriminada, restaurar el individualismo liberal y exonerar de toda responsabilidad social al derecho de propiedad privada (Borja, 2012, p. 1387).

2 Paradigma en el sentido kuhniano al que se le reconoce la posibilidad de éxito de resolver ciertos problemas agudos frente a otros competidores (Kuhn, 1971, pp. 51-52).

3 El aperturismo es una de las tesis planteadas por el neoliberalismo. Manifiesta una tendencia hacia la economía abierta, por un lado, dentro del Estado con la eliminación de trabas y controles a la producción e intercambio de bienes, y, por otro, fuera del Estado con la apertura de la economía a la inversión extranjera en igualdad de condiciones que la inversión nacional. Así como el abatimiento de barreras arancelarias y paraarancelarias para el ingreso o la salida de mercancías (Borja, 2012, p. 53). política. En ellos existe una percepción de menor corrupción con respecto a países que han asumido objetivos nacionalistas de recursos ${ }^{4}$, o poseen calificaciones satisfactorias frente a evaluaciones débiles de regímenes nacionalistas en la gobernanza energética (Transparencia Internacional, 2017; Natural Resource Governance Institute, 2017). En el afán de ser más competitivos, buscan cumplir estándares internacionales que involucran mayor fiscalización entre instituciones del Estado y transparencia de gobierno. Estos indicios permiten pensar la existencia de una relación causal entre liberalización petrolera y fortalecimiento en la rendición pública de cuentas.

Existen tres tipos de controles democráticos: vertical, horizontal y social. El control vertical o electoral es un "mecanismo de control ciudadano sobre los gobernantes" (Isunza y Gurza, 2015, p. 6). Los habitantes de un Estado retiran el poder o lo adjudican a un representante de modo que lo castiguen o recompensen mediante el voto; unido a esto, se suman indicadores de responsabilidad vertical que toman en cuenta aspectos relacionados con la competitividad política, la participación política y electoral, y la cultura política de los ciudadanos (O’Donnell, 1997, p. 144; Relly 2012, p. 336).

El control horizontal se ejerce por instituciones públicas que fiscalizan los actos del poder político y administrativo. Comprende actos que van "desde la fiscalización rutinaria hasta sanciones penales o destitución en rela-

4 Fontaine, Narváez y Fuentes (2019) ponen en evidencia la relación causal entre nacionalismo de petróleo y déficit de controles democráticos en países exportadores de petróleo de Latinoamérica. 
ción con actos u omisiones de otras instituciones del Estado" (O’Donnell, 1997, p. 155). El control social o vertical no electoral abarca movilizaciones sociales, grupos de protesta y denuncias frente a medios de comunicación (Ríos, Cortés, Suárez y Vélez, 2014, p. 281). Los medios adquieren relevancia porque permiten dar visibilidad a los problemas sociales y de quienes los atienden y rinden cuentas; a través del control social, los ciudadanos ejercen influencia sobre el sistema político y las burocracias de un Estado (Peruzzotti y Smulovitz, 2002, pp. 10-25).

El control democrático también implica la generación de transparencia, cuya relación con la liberalización se manifiesta a través de la competencia al proporcionar un incentivo fuerte para que los gobiernos permitan el libre acceso a la información como distintivo de mercados eficientes (Relly y Sabharwal, 2009, p. 148). Este incentivo hace que gobiernos apunten a organizaciones internacionales, que pueden otorgar beneficios al Estado si cumplen con estándares de gobernanza y transparencia. Los beneficios de esta competitividad pueden hacer económicamente más eficientes las instituciones de la política sectorial y mejorar a la par la transparencia en el área.

De acuerdo con las características teóricas expuestas, y la relación causal de la cual se presume su existencia, la pregunta de investigación es ¿por qué la liberalización del sector petrolero fortalece los controles democráticos en la política sectorial? La hipótesis que responde esta pregunta es que el Gobierno acoge explícitamente objetivos de liberalización para orientar el desarrollo a través del mercado, esto conlleva una reforma en la industria petrolera y permite al Gobierno desconcentrar las agencias estatales de petróleo, orientándolas hacia reglas del mercado y dotándolas de mayor autonomía. El Gobierno facilita un estilo de implementación participativo y descentralizado, lo que fortalece la transparencia, el control social y fomenta la rendición de cuentas.

El artículo explica la relación causal entre liberalización de la industria petrolera y el fortalecimiento de los controles democráticos a partir de la exposición teórica de causa y efecto, con el fin de explicar esta relación en Colombia como caso de estudio. Resulta fundamental entender el contexto institucional matizado principalmente por la violencia, la transformación del país a un Estado social de derecho con la Constitución de 1991, y su inclusión en el paradigma neoliberal mediante el proceso denominado Apertura Económica de principios de los ańos noventa. Las secciones subsiguientes exponen la teoría, el marco analítico, el método, los resultados y las conclusiones en torno a la relación causal y al diseño institucional.

\section{ENFOQUE TEÓRICO Y MARCO ANALÍTICO}

La teoría neoinstitucional comprende a las instituciones políticas como "colecciones de reglas y rutinas interrelacionadas que definen acciones apropiadas en términos de relaciones entre roles y situaciones" (March y Olsen, 1989, p. 160). Estas reglas y rutinas institucionalizan la acción de los individuos según las cuales es posible moldear su comportamiento (March y Olsen, 2010, pp. 135-136; Lowndes y Roberts, 2013, p. 179). Bajo estos asertos, 
la formulación de políticas se entiende como un proceso institucional (Peters, 2018) dado que las instituciones, en el sentido planteado, influyen en el diseño de las políticas. El análisis neoinstitucional de las políticas públicas del que parte esta investigación hace factible tratar a la política pública como el resultado de la arquitectura institucional de una sociedad (Eslava, 2010, p. 112).

Por otro lado, el marco analítico del diseño de política se basa en la selección de formas de intervención de un problema público a través de instrumentos, para crear representaciones y convertirlas en realidades (Peters, 2018, p. 5; Bobrow, 2006, p. 76). Se asume este marco porque comprende a los instrumentos de política pública como la sustancia del diseño de políticas, mediante los cuales es posible entender la acción pública al adecuar los medios a los fines de una política a través de la combinación instrumental (Howlett, Ramesh y Pearl, 2009, p. 114; Salamon, 2002; Lascoumes y Simard, 2011; Romero y Fontaine, 2017, p. 62).

Christopher Hood (1986) planteó una taxonomía de los instrumentos del Gobierno sintetizada en el esquema NATO. Nodalidad es la información que produce y recibe el gobierno; Autoridad son las normas jurídicas; Tesoro se refiere al presupuesto, las asignaciones y los recursos económicos; Organización representa las agencias formales con capacidad de acción directa (Fontaine, 2015, p. 83; Hood, 1986, p. 5). Esta propuesta es exhaustiva y útil porque los cuatro tipos de instrumentos se asumen como un tipo de evidencia (Lascoumes y Le Gales, 2007, p. 2), además, posibilitan ordenar los observables empíricos según las entidades del mecanismo causal.

\section{ONTOLOGÍA, METODOLOGÍA Y MÉTODO}

La presente investigación se posiciona en una ontología filosófica dualista al aceptar la existencia de una brecha entre mente y mundo que debe ser unida a través de comprobaciones empíricas de la realidad. Se asume una postura ontológica científica transfactual, en la que el conocimiento no está limitado a la observación directa, sino que se reconoce la presencia de hechos que, a priori, no están al alcance de la percepción sensible (Jackson, 2016, p. 40). Acorde con el principio de alineación 5 (Hall, 2003), la metodología subyacente de las ontologías asumidas es el realismo, porque trata la causalidad como la activación de poderes causales, tanto de objetos como de relaciones, para descubrir qué mecanismos hipotéticos o imaginarios no son imaginarios sino reales (Sayer, 2010, pp. 104-105; Bhaskar, 1998, p. 50).

El método que se alinea con la metodología realista es el seguimiento de procesos o process tracing, el cual trata la causalidad como mecanismo causal. Es un método de enfoque único que estudia el desarrollo de un mecanismo causal dentro de un caso, realizando inferencias sin proveer generalizaciones. Es factible trabajar el seguimiento de procesos en el diseño de política porque posibilita la utilización de los instrumentos como "unidades empíricamente observables" (Romero y Fontaine, 2017, p. 66). El rastreo de procesos identifica el mecanismo causal al examinar

5 La alineación es importante porque la idoneidad de un conjunto de métodos para solventar un problema determinado se basa en "suposiciones sobre la naturaleza de las relaciones causales que deben descubrir" (Hall, 2003, p. 374). 
TABLA 1. MECANISMO CAUSAL

\begin{tabular}{|c|c|c|c|c|c|}
\hline Teorización & $\begin{array}{c}\text { Adopción de objetivos } \\
\text { de política }(T)\end{array}$ & $\begin{array}{c}\text { Formulación de la } \\
\text { política (A) }\end{array}$ & $\begin{array}{c}\text { Coordinación } \\
\text { interinstitucional (B) }\end{array}$ & $\begin{array}{l}\text { Interacciones } \\
\text { políticas (C) }\end{array}$ & $\begin{array}{c}\text { Resultado } \\
\text { de la política (0) }\end{array}$ \\
\hline Operacionalización & $\begin{array}{l}\text { Objetivos de política } \\
\text { basados en el merca- } \\
\text { do, la desregulación y la } \\
\text { apertura }\end{array}$ & $\begin{array}{l}\text { Reforma petrolera hacia } \\
\text { la liberalización }\end{array}$ & $\begin{array}{l}\text { Políticas sectoriales orien- } \\
\text { tadas hacia la mercantili- } \\
\text { zación, el emprendimien- } \\
\text { to y la eficiencia }\end{array}$ & $\begin{array}{l}\text { Interacciones con actores } \\
\text { no estatales gestionados } \\
\text { por acuerdos y respon- } \\
\text { sabilidades compartidas }\end{array}$ & $\begin{array}{l}\text { Área de política mejora la } \\
\text { transparencia y los con- } \\
\text { troles democráticos }\end{array}$ \\
\hline Hipótesis & $\begin{array}{l}\text { Gobierno acoge explí- } \\
\text { citamente objetivos de } \\
\text { liberalización }\end{array}$ & $\begin{array}{l}\text { Gobierno reforma la po- } \\
\text { lítica petrolera basada en } \\
\text { objetivo de liberalización }\end{array}$ & $\begin{array}{l}\text { Gobierno adopta un esti- } \\
\text { lo de política de autoridad } \\
\text { descentrada hacia afuera }\end{array}$ & $\begin{array}{l}\text { Gobierno ejerce un estilo } \\
\text { de implementación parti- } \\
\text { cipativoy descentralizado }\end{array}$ & $\begin{array}{l}\text { Fortalecimiento de con- } \\
\text { troles democráticos }\end{array}$ \\
\hline Hipótesis alternativa & $\begin{array}{l}\text { Gobierno no acoge explí- } \\
\text { citamente objetivos de } \\
\text { liberalización }\end{array}$ & $\begin{array}{l}\text { Gobierno no reforma } \\
\text { la política petrolera ba- } \\
\text { sada en objetivo de } \\
\text { liberalización }\end{array}$ & $\begin{array}{l}\text { Gobierno no adopta un } \\
\text { estilo de política de auto- } \\
\text { ridad descentrada hacia } \\
\text { afuera }\end{array}$ & $\begin{array}{l}\text { Gobierno no ejerce un } \\
\text { estilo de implemen- } \\
\text { tación participativo y } \\
\text { descentralizado }\end{array}$ & $\begin{array}{l}\text { Debilitamiento de contro- } \\
\text { les democráticos }\end{array}$ \\
\hline
\end{tabular}

Fuente: basado en el mecanismo causal teórico desarrollado por Fontaine et al. (2018).

sistemáticamente las evidencias halladas a la luz de preguntas e hipótesis de investigación. Aunque estos mecanismos son difíciles de observar, indican con precisión cómo $\mathrm{C}$ transmite fuerzas causales para producir $\mathrm{O}$ en condiciones específicas (Collier, 2011, p. 823; Beach y Pedersen, 2013, p. 25; Capano y Howlett, 2019, p. 5).

El mecanismo causal (tabla 1) se plantea a nivel de teorización de acuerdo con la propuesta de Fontaine, Narváez y Fuentes (2018), que evidencia la transferencia de fuerzas causales desde la causa o detonante $(\mathrm{T})$ hasta el resultado $(\mathrm{O})$ :

El rastreo de procesos se complementa con la aplicación de una epistemología probabilística bayesiana. Contiene una carga subjetiva al postular que las probabilidades son creencias sobre una hipótesis lógicamente propuesta de acuerdo con la evidencia dada en combinación con factores contextuales (Mesa, Rivera y Romero, 2011; Fairfield y Charman, 2016; Befani y Mayne, 2014, p. 22). Apunta a evaluar el grado de probabilidad de una afirmación, por ello, el hallazgo de evidencias hace factible "aumentar el grado de confianza para cada relación causal única, basada en información imperfecta" (Paz y Fontaine, 2018, p. 6). La investigación parte de una probabilidad previa de $50 \%$ de que la hipótesis sea verdadera y $50 \%$ de que sea falsa; según las evidencias halladas y evaluadas, la probabilidad posterior aumentará o disminuirá, para confirmar o rechazar la hipótesis de investigación.

\section{SELECCIÓN DE CASO}

La selección de Colombia en el periodo 20022018 como caso de estudio se determinó mediante análisis comparado cualitativo (QCA). Esta técnica contempla la utilización de álgebra booleana $^{6}$, teoría de probabilidades (Schneider

6 El álgebra booleana fue desarrollada por George Boole en 1847. Se distingue del algebra aritmética al crear un producto lógico a partir de condiciones causales, mas no de valores que reproduzcan una operación matemática para obtener un resultado aritmético. Por ejemplo, una suma aritmética postula que $1+1$ es igual a 2 . En cambio, el álgebra booleana postula que $1+1$ es igual a 1 (Ragin, 
y Rohlfing, 2013, p. 561; Boole, 1854), uso de datos binarios y construcción de tabla de verdad. Al emplear álgebra booleana, el QCA admite la congregación de casos en conjuntos específicos representados en la tabla.

La agrupación de conjuntos es viable según la membresía a la que se adscriben, determinada en este trabajo por los atributos teóricos del detonante y del resultado. La membresía se evalúa al emplear variables dicotómicas definidas en conjuntos nítidos (crisp set, cs: 0 y 1), o pluricotómicas contenidas en conjuntos difusos (fuzzy sets, fs: entre 0 y 1), que connotan ausencia o presencia, tanto de la causa como del resultado (Schneider y Rohlfing, 2013).

La población de casos está conformada por ocho países con reservas y producción significativas de petróleo en Latinoamérica, según los parámetros definidos en la Revisión Estadística de Energía Mundial de British Petroleum, en el periodo 2007-2017: Argentina, Brasil, Colombia, Ecuador, México, Perú, Trinidad y Tobago y Venezuela. Los atributos teóricos de la liberalización petrolera persiguen la atracción de inversiones y el desarrollo a través del mercado (Michot, Ferrell y Volkov, 2007; Fontaine, Medrano y Narváez, 2019; Walter, 2016, p. 2). Estos atributos se operacionalizan a través de: 1) propiedad de la soc (X1), 2) tipo de regulación de la soc (X2), 3) régimen contractual (X3), y 4) creación de agencia reguladora nacional (X4).

1987, p. 89), es decir, si existe la presencia de dos condiciones causales, el producto será la presencia del resultado que se planteó a priori si la probabilidad de que existan esas condiciones causales es cierta.
Los atributos teóricos del resultado se conforman por controles horizontal, vertical, social y transparencia (O’Donnell, 1997; Peruzzotti y Smulovitz, 2002; Fox, 2007). La evaluación de los atributos propuestos se realiza a través de indicadores de gobernanza mundial en recursos extractivos hasta 2017. El indicador de Control de la Corrupción del Worldwide Governance Indicators (WGI) ilustra el control horizontal (Y1). La posición de los países en el índice desarrollado por el Natural Resource Governance Institute (NRGI) define el control social (Y2). Por último, la transparencia se evalúa mediante la Iniciativa de Transparencia de las Industrias Extractivas (EITI) (Y3), cuya ratificación simboliza la voluntad del Gobierno para promover la transparencia en los ingresos, monitorear el cumplimiento de contratos, asegurar que las empresas publiquen lo que pagan y que los gobiernos divulguen lo que reciben de la extracción de recursos naturales (Fontaine et al., 2019, p. 76; Mejía, 2013, p. 92).

\section{TABLA DE VERDAD Y TIPOLOGÍA DE CASOS}

Los atributos de detonante y resultado se evaluaron a través de los conjuntos que se presen$\tan$ en la tabla 2.

La información de X1 se obtuvo de leyes de hidrocarburos, estatutos de soc y bolsas de valores vigentes al 2018. Los datos representados en X2, X3 y X4 constan en la investigación sobre desempeño de instituciones en el sector petrolero latinoamericano de Balza y Espinasa hasta el 2015, así como en leyes de hidrocarburos y estados financieros al 2018. Los valores 
TABLA 2. CS Y FS QCA

\begin{tabular}{|c|c|c|c|c|c|c|c|c|c|c|}
\hline \multirow{2}{*}{ Población de casos } & \multicolumn{5}{|c|}{ Liberalización petrolera (X) } & \multicolumn{4}{|c|}{ Controles democráticos $(\mathrm{Y})$} & \multirow{2}{*}{$X^{*} \mathbf{Y}$} \\
\hline & $\mathrm{X} 1$ & $\mathrm{X} 2$ & $\mathbf{X 3}$ & $\mathrm{X} 4$ & $\mathrm{X} 1+\mathrm{X} 2+\mathrm{X} 3+\mathrm{X} 4 / 4$ & Y1 & Y2 & Y3 & $\mathrm{Y} 1+\mathrm{Y} 2+\mathrm{Y} 3 / 3$ & \\
\hline Argentina & 1 & 0 & 0 & 0 & 0,25 & 0 & 0 & 0 & 0,00 & 0,00 \\
\hline Brasil & 1 & 1 & 1 & 1 & 1,00 & 0 & 1 & 0 & 0,33 & 0,33 \\
\hline Colombia & 1 & 1 & 1 & 1 & 1,00 & 0 & 1 & 1 & 0,67 & 0,67 \\
\hline Ecuador & 0 & 0 & 0 & 0 & 0,00 & 0 & 0 & 0 & 0,00 & 0,00 \\
\hline México & 0 & 0 & 0 & 0 & 0,00 & 0 & 1 & 1 & 0,67 & 0,00 \\
\hline Perú & 1 & 1 & 0,5 & 1 & 0,88 & 0 & 1 & 1 & 0,67 & 0,58 \\
\hline Trinidad y Tobago & 1 & 1 & 1 & 0 & 0,75 & 0 & 1 & 1 & 0,67 & 0,50 \\
\hline Venezuela & 0 & 0 & 0 & 0 & 0,00 & 0 & 0 & 0 & 0,00 & 0,00 \\
\hline \multicolumn{6}{|c|}{$\mathrm{X} 1$ : Inversión privada directa $=1 ;$ Monopolio estatal $=0$} & \multirow{2}{*}{\multicolumn{5}{|c|}{ Y1: Promedio control de la corrupción: $-2,5$ a $0=0 ; 0,01$ a $2,5=1$}} \\
\hline \multicolumn{6}{|c|}{$\mathrm{X} 2$ : Derecho privado $=1$; Derecho público $=0$} & & & & & \\
\hline \multicolumn{6}{|c|}{ X3: Contrato de concesión $=1$; Concesión para el midstream $=0,5$; Contrato de servicios $=0$} & \multicolumn{5}{|c|}{ Y2: Mayor a $60=1$; Menor a $60=0$} \\
\hline \multicolumn{6}{|c|}{ X4: Creación agencia reguladora $=1 ;$ No existe agencia reguladora $=0$} & \multicolumn{5}{|c|}{ Y3: Miembro $=1 ;$ No miembro $=0$} \\
\hline \multicolumn{11}{|c|}{ Valores en la conjugación de detonante y resultado ( $\left.X^{*} Y\right)$ : Entre 0 a 0,49 representan "ausencia". Entre 0,50 a 1 simbolizan "presencia". } \\
\hline
\end{tabular}

de $\mathrm{Y} 1$ representan un promedio por país, desde 1996 a 2017, en el cual el rango varía de -2,5 (débil) a 2,5 (fuerte) según WGI. Las cifras de Y2 constan en el índice del NRGI hasta 2017; si la estimación es mayor a 60, el país tiene una gobernanza buena y satisfactoria, si es menor a 60, la gobernanza es débil, deficiente o mala. La condición de miembro de EITI en Y3 consta en el estándar de la Iniciativa al 2018. Finalmente, los valores de Xy Y se obtuvieron mediante un promedio de sus atributos.

La combinación de causa y resultado mediante una multiplicación de X y Y, siguiendo la propuesta de Fontaine et al. (2019), arroja los tipos de casos según la consistencia que mantienen con la hipótesis de investigación (tabla 3).

Colombia, Perú y Trinidad y Tobago cumplen con la causa y también con el resultado. De estos tres países se escoge Colombia como caso de estudio porque expone el valor más alto en la tabla de verdad $(0,67)$, además, su contexto está determinado por un cambio institucional ejemplar en Latinoamérica derivado
TABLA 3. TIPOLOGÍA DE CASOS

\begin{tabular}{lll}
\hline & \multicolumn{2}{c}{ Liberalización petrolera } \\
\hline Fortalecimiento de controles & \multicolumn{1}{c}{+} & \multicolumn{1}{c}{-} \\
democráticos & Típicos & Inconsistente \\
& Colombia & México \\
& Perú & \\
& Trinidad y Tobago & \\
\cline { 2 - 3 } & Desviante & Individualmen- \\
& Brasil & te irrelevantes \\
& & Argentina \\
& & Ecuador \\
& & Venezuela \\
\hline
\end{tabular}

Fuente: elaboración propia a partir de Schneider y Rohlfing (2013).

de la adopción de una nueva Constitución y la entrada en vigencia de la Apertura Económica en 1991. El periodo de estudio comprende del 2002 al 2018, en los gobiernos de Uribe y Santos, primero porque corresponde a un cambio notable en la política petrolera, y segundo, porque la extensión abarcada permite analizar la implementación de la política hasta un resultado empíricamente comprobable. El mecanismo causal propuesto para Colombia puede aplicarse a Perú y Trinidad y Tobago en investigaciones posteriores, a manera de comprobación de la teoría. 


\section{PRUEBAS EMPÍRICAS}

Según los grados de certeza y exclusividad, existen cuatro tipos de pruebas empíricas: hoop test, smoking gun test, doubly decisive test y straw in the wind test (van Evera, 1997, pp. 31-32). El tipo de prueba utilizado es hoop test o prueba del aro, porque define un estándar más exigente e introduce un criterio necesario, pero no suficiente, para aceptar la explicación (Ramírez, Narváez y Fontaine, 2018, p. 28; Bennett, 2010, p. 210). Las otras pruebas se descartan por su debilidad, demanda de recursos o deficiencia en la calidad de la investigación.

En la tabla 4 se presentan pruebas del aro diseñadas por cada entidad del mecanismo causal.

\section{RESULTADOS}

El método permite corroborar la hipótesis general del trabajo al hallar evidencias conforme a las subhipótesis planteadas en las pruebas

TABLA 4. PRUEBAS EMPÍRICAS DEL ARO

\begin{tabular}{|c|c|c|}
\hline Hipótesis para el Detonante (HT) & Instrumento & Evidencia empírica esperada \\
\hline \multirow{4}{*}{$\begin{array}{l}\text { Gobierno acoge explícitamente objetivos de } \\
\text { liberalización }\end{array}$} & Nodalidad & Intenciones declaradas del Gobierno en programas político administrativos \\
\hline & Autoridad & Intenciones explícitas del Gobierno en el marco normativo \\
\hline & Tesoro & Intenciones del Gobierno reflejadas en el desarrollo orientado al mercado \\
\hline & Organización & Intenciones del gobierno manifestadas en el nuevo diseño de las agencias estatales \\
\hline Hipótesis para la Entidad A (HA) & Instrumento & Evidencia empírica esperada \\
\hline \multirow{4}{*}{$\begin{array}{l}\text { Gobierno reforma la política petrolera basada en } \\
\text { objetivo de liberalización }\end{array}$} & Nodalidad & Planes y documentos administrativos de la política sectorial orientados a la apertura \\
\hline & Autoridad & Reformas en el marco normativo concerniente al área de política \\
\hline & Tesoro & $\begin{array}{l}\text { Apertura a la inversión privada mediante capitalización por acciones en la política } \\
\text { sectorial }\end{array}$ \\
\hline & Organización & Modificación institucional de las agencias del Estado dedicadas al petróleo \\
\hline Hipótesis para la Entidad B (HB) & Instrumento & Evidencia empírica esperada \\
\hline \multirow{4}{*}{$\begin{array}{l}\text { Gobierno adopta un estilo de política de autoridad } \\
\text { descentrada hacia afuera }\end{array}$} & Nodalidad & Planificación intersectorial con incidencia del mercado \\
\hline & Autoridad & Marco normativo facilita la inversión privada en bienes y servicios públicos \\
\hline & Tesoro & Impulso multisectorial a la inversión privada \\
\hline & Organización & $\begin{array}{l}\text { Cambios en instituciones de la administración pública para dotarlas de autonomía y } \\
\text { favorecer la participación privada }\end{array}$ \\
\hline Hipótesis para la Entidad C (HC) & Instrumento & Evidencia empírica esperada \\
\hline \multirow[t]{4}{*}{$\begin{array}{l}\text { Gobierno ejerce un estilo de implementación parti- } \\
\text { cipativo y descentralizado }\end{array}$} & Nodalidad & $\begin{array}{l}\text { Modalidades de acceso a la información para actores no estatales por parte de la } \\
\text { empresa estatal de petróleo }\end{array}$ \\
\hline & Autoridad & Normativa fomenta acuerdos mutuos y consultas previas \\
\hline & Tesoro & Mayor participación y aprovechamiento de las rentas petroleras por las localidades \\
\hline & Organización & Fortalecimiento institucional de entes territoriales que reciben regalías \\
\hline Hipótesis para el Resultado (HO) & Instrumento & Evidencia empírica esperada \\
\hline \multirow{4}{*}{$\begin{array}{l}\text { Fortalecimiento de controles democráticos en la } \\
\text { política petrolera }\end{array}$} & Nodalidad & Libre acceso a información pública sobre la actividad petrolera \\
\hline & Autoridad & Respeto al debido proceso \\
\hline & Tesoro & Reportes financieros oportunos y transparentes \\
\hline & Organización & Independencia de las agencias de evaluación y control \\
\hline
\end{tabular}

Fuente: elaboración propia. 
empíricas. El procedimiento del rastreo de procesos sucede cuando se postula que: si lo que se plantea en la subhipótesis de cada entidad del mecanismo causal es cierto, ¿qué se espera encontrar en instrumentos NATO? La evidencia empírica esperada orienta el hallazgo de evidencias en función de los atributos teóricos propuestos para detonante y resultado.

En relación con esto, el rastreo de procesos guía hacia evidencias que comprueban la hipótesis, y aunque puedan existir gran cantidad de evidencias, a continuación se presentan aquellas que en conjunto son necesarias pero no suficientes para aceptar cada subhipótesis de acuerdo con la prueba del aro. La hipótesis alternativa se rechaza en función de las huellas o evidencias que cumplen con los criterios de explicación de la hipótesis. Si no existen, es válido aceptar la hipótesis alternativa, lo que se refleja en el valor de la confianza posterior como se verá en el instrumento de organización de entidad $\mathrm{O}$.

\section{Detonante: renovación de la administración pública}

Las intenciones de liberalización del Gobierno se hallaron en el Plan Nacional de Desarrollo (PND) 2002-2006, y en el Programa de Renovación de la Administración Pública (PRAP), contenido en la Directiva Presidencial 10 de 2002. En ambos casos, el Gobierno manifestó su voluntad de reformar la administración pública para conseguir un Estado gerencial, eficiente y austero con los recursos públicos, reducir el gasto público y fomentar la descentralización estatal y la autonomía regional (DNP, 2003, p. 19). Se explicitó mayor parti- cipación privada en asuntos estatales al seńalar el alistamiento de procesos de concesiones en los servicios públicos domiciliarios.

La disposición del marco normativo hacia la liberalización se halló en los artículos 1 y 8 de la Ley 812 de 2003, que dispusieron el acceso de la economía nacional a los mercados financieros, de bienes y servicios internacionales, y la ejecución de programas de inversión destinados a impulsar la exploración y explotación de hidrocarburos mediante la promoción gubernamental de inversión privada nacional y extranjera en exploración y perforación. Además, se buscó suscribir, en promedio anual, 20 contratos de asociación, estudiar nuevos esquemas contractuales en el sector de hidrocarburos y avanzar en la implementación del "Plan maestro para la refinería de Cartagena", utilizando para ello la mayor inversión privada posible y financiamiento parcial de la enajenación de inversiones no estratégicas de Ecopetrol.

A nivel del tesoro, las evidencias contenidas en el numeral 1.1.2 del PRAP, PND y en los artículos 1 y 19 de la Ley 790 de 2002 demuestran la preferencia del Gobierno por el desarrollo orientado al mercado. Estas evidencias dan cuenta de que el Gobierno previó reducir el costo del aparato estatal, de la burocracia y de los privilegios de funcionarios de alto nivel. Las directrices de austeridad exigieron que los jefes de entidades del orden nacional racionalicen el personal de libre nombramiento y remoción, eliminen vacantes, restrinjan horas extras, entre otros. Para racionalizar el uso de recursos públicos, la Ley 790 facultó al Gobierno para poner límites a gastos de funcionamiento de corporaciones autónomas regionales (CAR) y 
de corporaciones de desarrollo sostenible. En el PND, los subsidios a los combustibles fueron señalados por el Gobierno como restrictivos para el desarrollo de un mercado competitivo en el sector petrolero, para ello, se propuso adoptar un sistema general de precios acorde con la realidad de los mercados internacionales como una señal de estabilidad para los inversionistas.

Las intenciones del Gobierno de abrirse al mercado mediante un rediseño de las instituciones públicas se comprobaron mediante el artículo 16 de la Ley 790, que invistió al presidente de facultades extraordinarias temporales para liderar una reforma administrativa en las agencias estatales, con lo cual realizó supresiones, fusiones o escisiones en organismos del Estado, entre ellas, agencias del petróleo. Por su parte, el PND y el artículo 1 de la Ley 812-2003, persiguieron la reestructuración de empresas de servicios públicos cuya viabilidad económica sea incierta, y apuntaron a incrementar la eficiencia del Estado mediante el rediseño de las entidades nacionales y el fortalecimiento de la descentralización y la autonomía territorial.

\section{Entidad A: reforma petrolera hacia el aperturismo y la desregulación}

Planes y documentos administrativos de la política petrolera demostraron que a raíz de la reforma se potenció una mayor competencia entre empresas petroleras. Varias compañías con presencia histórica en Colombia fortalecieron su posición y otras nuevas entraron o regresaron al país, como Occidental, Chevron, BP, Burlington, ExxonMobil, Petrobras, Repsol YPF, Perenco, entre otras. Asimismo, el eje 2 del plan de inversiones de Ecopetrol y el "Prospecto de información constitutivo del programa de emisión y colocación de acciones" apuntaron a la internacionalización de la empresa para evitar que la explotación se realice solo en Colombia mediante contratos de asociación, sino que sea ejecutada en forma directa. La ampliación exploratoria se realizó a través de la compra de reservas, la adquisición de activos exploratorios, la exploración en bloques en otros países, dirigiéndose hacia Latinoamérica y el golfo de México.

Las reformas en el marco normativo para connotar la apertura de la industria hacia reglas de mercado se evidenciaron en el numeral 4.2.3 del PND 2006-2010, que informó la transición de un contrato de asociación para la exploración y producción a uno de concesión moderna, donde Ecopetrol participe como un agente de mercado en igualdad de condiciones con otras operadoras petroleras públicas o privadas. El Decreto-Ley 1760-2003 dispuso que las funciones de Ecopetrol deben ejercerse según lo previsto en el Código de Comercio, y el artículo 6 de la Ley 1118 de 2006 evidenció la desregulación de la industria nacional al definir como régimen aplicable a Ecopetrol, exclusivamente, el derecho privado.

El artículo 1 de la Ley 1118 organizó a Ecopetrol como una sociedad de economía mixta, de carácter comercial; gracias a ello, en el artículo 8 de los estatutos sociales de Ecopetrol, contenidos en la escritura pública 5314 del 14 de diciembre de 2007, se definió la emisión de acciones de acuerdo con el capital autorizado. Concomitantemente, Ecopetrol emprendió un programa para capitalizar la empresa con la colocación de al menos el $10 \%$ más una acción del capital de la compañía. El 
programa de colocación y emisión de acciones tuvo el objetivo de alcanzar la mayor participación posible de inversionistas privados y acceder a los beneficios de un régimen de sociedad de economía mixta. Se contemplaron tres rondas de emisión de acciones ordinarias, tan solo en la primera oferta de la Ronda 1 se ofertaron 4'087.723.771 acciones, equivalentes a $\$ 5722.813 .279 .400$ de acuerdo con un precio de suscripción de $\$ 1400$ por acción.

La modificación de la arquitectura organizacional en la política petrolera se realizó mediante el artículo 1 del Decreto-Ley 1760 de 2003 que escindió Ecopetrol, separando sus funciones operativas y reguladoras. La división contempló la desvinculación de Ecopetrol de la administración de las reservas de hidrocarburos propiedad de la nación y la administración de los activos no estratégicos representados en acciones y participaciones en sociedades. A través de los artículos 2 y 5 de la misma ley, se creó una entidad administrativa estatal denominada Agencia Nacional de Hidrocarburos (ANH), que asumió las funciones regulativas retiradas a Ecopetrol, particularmente en la administración de las áreas hidrocarburíferas del país, asignación para su exploración y explotación, y apoyo al ministerio del ramo en la formulación de la política pública y los planes sectoriales.

\section{Entidad B: un nuevo estilo de política}

La información sobre la orientación hacia la mercantilización de políticas sectoriales adyacentes a la petrolera consta en los documentos Conpes 3281 y 3385, que manifestaron una estrategia de enajenación de participaciones de la nación y participación privada en servi- cios públicos domiciliaros, respectivamente. En 2004, el Gobierno propuso una estrategia de enajenación de activos públicos y participaciones del Estado en general cuyo objetivo fue reducir el déficit fiscal, democratizar la propiedad accionaria para construir un "país de propietarios" y fortalecer el mercado de capitales. Para este cometido, se dispuso que las financieras de la nación ofrezcan líneas de crédito y dispongan de canales de distribución para la oferta de acciones al público en general. Estos documentos determinaron lineamientos de política a nivel sectorial para admitir capital privado en los siguientes sectores: 1) agua potable y saneamiento básico, 2) eléctrico, 3) gas, 4) telecomunicaciones.

El marco normativo facilitó la inversión privada en bienes y servicios públicos mediante la captación de inversiones y la conformación de alianzas público/privados. En el primer caso, se emitió la Ley 963 que contempló el establecimiento de contratos de estabilidad jurídica para promover nuevas inversiones y ampliar las existentes. El artículo 2 de esta ley señaló como beneficiarios a inversionistas en los sectores de turismo, minería, petróleo, telecomunicaciones, agricultura, infraestructura, entre otros. En el segundo caso, la Ley 1508 definió el régimen jurídico de asociaciones público privadas (APP) para la provisión de bienes y servicios públicos, que tuvieron lugar en los contratos entre una entidad del Estado y una persona natural o jurídica de derecho privado, en donde los mecanismos de pago se definieron de acuerdo con el grado de calidad de la infraestructura o servicio provisto.

En el tesoro se impulsó la inversión privada en varios sectores de política mediante el 
"Programa de apoyo al proceso de participación privada y concesiones en infraestructura", cuya inversión en el PPCI-2 (2004-2008) fue de US\$7'150.000 de los cuales el Banco Interamericano de Desarrollo (BID) aportó 5 millones y el Estado el resto ${ }^{7}$. El éxito del programa permitió aplicar el PPCI-3 (2008-2013) donde la inversión aumentó a US\$9'750.000 de los cuales el BID entregó 7 millones (BID, 2018, p. $98)^{8}$. Además, se registró un ascenso significativo en la inversión privada en infraestructura de tecnologías de la información y la comunicación (TIC), transporte, minas, energía y agua, pasando de \$6127.551 millones en 2002 a \$24'113.887 millones en 2011 (DNP, s. f.).

Mediante el Consejo Nacional de Política Económica y Social (Conpes 3281) se conformó un Comité de Aprovechamiento de Activos Públicos y se encargó al Fondo Financiero de Proyectos de Desarrollo (FONADE) la administración de acciones y la ejecución de los procesos de enajenación. El proceso aterrizó con la enajenación de la propiedad estatal de Ecogás a favor de Transportadora de Gas del Interior, y la enajenación del 99,96\% de acciones que el Fondo de Garantías de Instituciones Financieras poseía en Granahorrar Banco Comercial S. A. La autonomía se consolidó con la reforma al Departamento Nacional de Planeación (DNP) mediante Decreto 3517-2009, cuyo nuevo rol fue coordinar la autonomía de varias agencias públicas, especialmente en la estructuración, coordinación y ejecución de los procesos de participación privada en infraestructura, trans-

7 Contrato de préstamo BID I 594/OC-CO.

8 Contrato de préstamo BID 2079/OC-CO. porte, minas, hidrocarburos, energía y en los servicios de telecomunicaciones.

\section{Entidad C: implementación participativa y descentralizada}

La empresa estatal de petróleo se obligó a la atención de consultas públicas de información, así lo demuestran las resoluciones 08 y 17 de 2005 que crearon la Oficina de Participación Ciudadana y materializaron las responsabilidades de Ecopetrol para atender consultas de interés público en materia petrolera. Estas condiciones, creadas en 2005, rindieron frutos para que en 2009 la participación y atención de información aumentara a varias localidades. No solo con oficinas de participación ciudadana, sino también para cubrir regiones del país donde aún no era posible la atención directa, lo que se logró colocando sistemas de líneas gratuitas (teleiguanas) y facilitando brigadas móviles para atención a requerimientos sobre las operaciones petroleras (Ecopetrol, 2009, p. 89).

En autoridad se hallaron acuerdos mutuos y consultas previas como formas de admitir la participación y fomentar una cogobernanza. Las evidencias constan en los decretos 2893-2011 y 2613-2013 que adjudicaron la ejecución de consultas previas al Ministerio del Interior. Ligado a ello, el poder judicial propició el acceso a la consulta previa según consta en las sentencias de la Corte Constitucional T-730 de 2016 y SU-123 de 2018, que beneficiaron a las comunidades indígenas de Alto Lorenzo y Awá La Cabańa frente a la extracción petrolera. Por último, los acuerdos mutuos se manifestaron en las convenciones 
colectivas de trabajo 2009-2014 y 2014-2018 entre la Unión Sindical Obrera (uso) y Ecopetrol, que históricamente mantuvieron una relación violenta. Al propiciar el acuerdo y la participación, se allanó el camino para nuevas interacciones entre el sector petrolero y los sindicatos con base en acuerdos pacíficos.

Una mayor participación de localidades en las rentas petroleras se detectó en las evoluciones positivas de inversión social, distribución de impuestos y pago de regalías a comunidades. En primer lugar, se incrementó la inversión social de Ecopetrol, que pasó de \$34.392 millones en 2005 a \$341.560 millones en 2013 (Ecopetrol, 2009, p. 79; 2014, p. 313). Segundo, la distribución de los impuestos de Ecopetrol a entidades territoriales pasó de $\$ 176.933$ millones en 2011 a $\$ 298.884$ millones en 2015 (Ecopetrol, 2012, pp. 94-95; 2016, pp. 217-218). Por último, aumentaron las regalías ${ }^{9}$ por producción de petróleo pagadas por departamento, no solo de Ecopetrol, sino de toda la producción petrolera en general, que subió de \$1'393.363.857 millones en

9 Aunque en diez años las regalías aumentaron por departamento, evaluaciones posteriores a la reforma del Sistema General de Regalías (SGR) (Martínez, 2016; Bonet y Urrego, 2014) evidenciaron también un pobre aprovechamiento por parte de las comunidades e insostenibilidad de los proyectos. Las evidencias en este instrumento confirman la subhipótesis que trata sobre la mayor participación en el tesoro, algo que se reconoce en diagnósticos acerca del SGR después de su reforma (Bonet y Urrego, 2014, p. 40), y aunque el aprovechamiento no fue considerado, es relevante dejar esta puerta abierta para investigaciones futuras que clarifiquen la relación entre mayor participación en regalías y mejor aprovechamiento.
2004 a \$15’472.498.590 millones en 2014 (Asociación Colombiana del Petróleo, 2019). Los entes territoriales beneficiarios de regalías se empoderaron gracias al proyecto de fortalecimiento institucional de los municipios petroleros llevado a cabo entre Ecopetrol y la Corporación Financiera Internacional del Grupo Banco Mundial, favoreciendo a los municipios de Acacías, Maní, Yopal, Purificación y de Aipe (Ecopetrol, 2009, p. 88). Asimismo, en el Acto Legislativo 05 de 2011 y en la Ley 1530 de 2012 se modificaron los artículos 360 y 361 de la Constitución para que los ingresos del Sistema General de Regalías (SGR), se destinen a financiar proyectos en entidades territoriales donde se adelanten explotaciones de recursos naturales no renovables. Además, se permitió que alcaldes y gobernadores conformen una Comisión Rectora del SGR, lo que garantizó las asignaciones directas en virtud de su derecho a participar en regalías y compensaciones.

\section{Resultado: aumento en los controles democráticos}

Con la aprobación de la Ley 1712 se garantizó la transparencia y el acceso a la información pública, además, los avances alcanzados por Colombia en el marco de la Alianza para el Gobierno Abierto materializaron el objetivo de transparencia con la creación del Portal del Estado Colombiano, la estrategia de Gobierno en Línea, la plataforma Mapa Regalías y la publicación de todos los procesos de contratación del Estado. En segundo lugar, la validación independiente del cumplimiento de los requisitos de Colombia para formar parte de 
EITI determinó un cumplimiento significativo, satisfactorio o sobresaliente de los mismos. Por último, la evidencia de que la información no solamente está disponible, sino que es utilizada efectivamente por los ciudadanos, consta en la tendencia positiva que reflejó Colombia dentro del indicador Voz y Responsabilidad del wGI, que pasó de una estimación de -0,34 en 1998 a una valoración positiva de 0,11 en 2017 .

La institucionalización del debido proceso se evidenció en el fortalecimiento de las capacidades de la Fiscalía General de la Nación, cuyas investigaciones y acusaciones en materia de lucha contra la corrupción han tenido mayor efectividad para que la justicia sancione delitos como peculado, prevaricato, cohecho, enriquecimiento ilícito, entre otros (Hernández, 2018). Esta institucionalización consta en el incremento de sanciones penales entre 2008 y 2015 , que pasaron de alrededor de 150 a 496 sanciones penales por corrupción (sIRI y Procuraduría General de la Nación, en Hernández, 2018, p. 109). Asimismo, la evaluación de la efectividad práctica de marcos legislativos anticohecho aplicada a Colombia, dio como resultado un avance significativo en la aplicación de la Convención de las Naciones Unidas contra la Corrupción, con un porcentaje de avance de $91 \%$ en 2018 (Observatorio de Transparencia y Anticorrupción, s. f.).

En tesoro, se comprobó el cumplimiento del compromiso 11 del Plan de Acción 20152017 de Gobierno Abierto, según el cual Colombia se propuso fortalecer la transparencia y el control ciudadano en las regalías. El cumplimiento de este compromiso fue de grado "completo", a través de la creación del Mapa
Inversiones en 2015 hasta 2017. Además, dentro del estándar eiтr, se cumplieron los requisitos 4.1. y 4.5., que confirman la prueba empírica, pues el Estado colombiano cumplió, con un grado de progreso "satisfactorio", con la divulgación exhaustiva de impuestos e ingresos relacionados con la industria extractiva, además, Ecopetrol publicó la información necesaria sobre los aspectos exigidos por EITI (Cowater Sogema, 2018).

Las evidencias en organización sobre independencia de las agencias de evaluación y control son negativas. El indicador sobre efectividad del gobierno tiene un promedio de -0,12, el indicador de Estado de derecho refleja un promedio de $-0,47$, y el indicador sobre control de la corrupción demuestra un promedio de -0,29; mediciones realizadas entre 1998 a 2017. Subsiste una desinstitucionalización de varias agencias de control del Estado al ser vulnerables a la intervención del Ejecutivo, especialmente en el periodo de Uribe. Un ejemplo de ello es el Consejo Superior de la Judicatura, que fue cuestionado por tener excesiva influencia en la designación y control disciplinario de jueces y magistrados, lo que dio lugar a censura en sus providencias (Suárez, 2010, p. 152).

\section{Evaluación de evidencias con fórmula bayesiana}

De acuerdo con las evidencias, los test empíricos de $\mathrm{T}$ a $\mathrm{C}$ son positivos y poseen una confianza posterior $\mathrm{p}(\mathrm{h} / \mathrm{e})$ de 0,99 . Mientras que para $\mathrm{O}$, la prueba empírica en instrumento de organización es negativa, lo que reduce la confianza posterior a 0,79 ; sin embargo, este valor es suficiente para corroborar la hipóte- 
sis del resultado, porque es mayor al valor de la confianza inicial $\mathrm{p}(\mathrm{h})$ que partió con 0,50 (tablas 5 y 6).

TABLA 5. CONFIANZA POSTERIOR DE PRUEBAS EMPÍRICAS DE ENTIDAD T A ENTIDAD C

\begin{tabular}{lcccc}
\hline & Nodalidad & Autoridad & Tesoro & Organización \\
\hline $\mathrm{P}(\mathrm{h})$ & 0,5 & 0,75 & 0,9 & 0,96 \\
\hline $\mathrm{P}(\sim \mathrm{h})$ & 0,5 & 0,25 & 0,1 & 0,04 \\
\hline $\mathrm{P}(\mathrm{e} \mid \mathrm{h})$ & 0,9 & 0,9 & 0,9 & 0,9 \\
\hline $\mathrm{P}(\sim \mathrm{e} \mid \mathrm{h})$ & 0,1 & 0,1 & 0,1 & 0,1 \\
\hline $\mathrm{P}(\mathrm{e} \mid \sim \mathrm{h})$ & 0,3 & 0,3 & 0,3 & 0,3 \\
\hline $\mathrm{P}(\sim \mathrm{e} \mid \sim \mathrm{h})$ & 0,7 & 0,7 & 0,7 & 0,7 \\
\hline $\mathrm{P}(\mathrm{h} \mid \mathrm{e})$ & 0,75 & 0,9 & 0,96 & 0,99 \\
\hline
\end{tabular}

Fuente: adaptado de Fontaine et al. (2017).

TABLA 6. CONFIANZA POSTERIOR DE PRUEBA EMPÍRICA DE ENTIDAD O

\begin{tabular}{lcccc}
\hline & Nodalidad & Autoridad & Tesoro & Organización \\
\hline $\mathrm{P}(\mathrm{h})$ & 0,5 & 0,75 & 0,9 & 0,96 \\
\hline $\mathrm{P}(\sim \mathrm{h})$ & 0,5 & 0,25 & 0,1 & 0,04 \\
\hline $\mathrm{P}(\mathrm{e} \mid \mathrm{h})$ & 0,9 & 0,9 & 0,9 & 0,9 \\
\hline $\mathrm{P}(\sim \mathrm{e} \mid \mathrm{h})$ & 0,1 & 0,1 & 0,1 & 0,1 \\
\hline $\mathrm{P}(\mathrm{e} \mid \sim \mathrm{h})$ & 0,3 & 0,3 & 0,3 & 0,3 \\
\hline $\mathrm{P}(\sim \mathrm{e} \mid \sim \mathrm{h})$ & 0,7 & 0,7 & 0,7 & 0,7 \\
\hline $\mathrm{P}(\mathrm{h} \mid \mathrm{e})$ & 0,75 & 0,9 & 0,96 & 0,99 \\
\hline $\mathrm{P}(\mathrm{h} \mid \sim \mathrm{e})$ & 0,13 & 0,3 & 0,56 & $\mathbf{0 , 7 9}$ \\
\hline
\end{tabular}

Fuente: adaptado de Fontaine et al. (2017).

\section{CONCLUSIONES}

El análisis de la política petrolera a través del diseño demostró la preferencia de los tomadores de decisiones por unos instrumentos de cariz mercantilista y privatizador, evidencia de una apuesta ideológica liberal de los gobiernos de Uribe y Santos. Esta apuesta se manifestó en la definición del problema público y del objetivo de política, determinados bajo un enfoque economicista, lo que permite colegir que el cambio de política hacia el mercado es resultado tardío del cambio institucional en el que se sumergió Colombia en 1991 con la Apertura Económica y el cambio de Constitución. El nuevo diseńo institucional creó una cultura en los tomadores de decisiones en la cual expresan preferencias por instrumentos ligados a una gobernanza de mercado, Nueva Gestión Pública y alianzas público/privadas; elementos que se pusieron en marcha en la política petrolera como expresión general de su liberalización.

El fortalecimiento de los controles democráticos echó raíces a partir de la entidad C, donde consta un procesamiento de conflictos que da cabida al consenso, participación en la riqueza y consultas previas. Sin embargo, esta relación Estado-sociedad estuvo matizada por los intereses económicos de los tomadores de decisiones, que eligieron este modo de gobernanza por las oportunidades que le abrieron paso a la industria en su camino hacia la internacionalización. Lo que no significa que la política gozó de un diseño plural, al contrario, fue evidente la preponderancia del Gobierno iniciada en el ejercicio de facultades extraordinarias, así como la falta de autonomía de agencias de control, lo que genera la inquietud acerca de por qué en Colombia las instituciones no avanzan en un proceso de independencia. Una mayor participación en regalías no significa mejor aprovechamiento, el SGR mantiene una deuda al respecto. Esto allana el camino para investigaciones futuras sobre la importancia del rol de la sociedad civil en la construcción de la política petrolera para avanzar en la paz, la independencia de funciones, mayor redistribución y mejor aprovechamiento de la riqueza como una responsabilidad pública y ciudadana. 
Por último, quedó de manifiesto la influencia de iniciativas, estándares y alianzas internacionales dentro de la política petrolera. Las evidencias demuestran el posicionamiento de nuevos sujetos políticos que ejercieron particular influjo al momento de activar la voluntad de gobiernos por ser transparentes y rendir cuentas. La influencia sucedió en un escenario de dependencia estatal a estas iniciativas para conseguir metas bajo la venia de sujetos políticos supranacionales, que reclamaron a la industria petrolera una mínima atención a las demandas o aspiraciones de la sociedad civil y de gobiernos subnacionales. Esto puede significar la inauguración de políticas formuladas con mayor participación desde abajo, el involucramiento en los procesos del gobierno que mejoren la calidad de la ciudadanía, su empoderamiento de los asuntos públicos y un acercamiento hacia una auténtica poliarquía.

\section{REFERENCIAS}

Asociación Colombiana del Petróleo (2019). Informe Estadistico Petrolero 2019. Recuperado de https:// acp.com.co/web2017/es/publicaciones-e-informes/informe-estadistico-petrolero/271-informeestadistico-petrolero-actualizado-marzo.

Balza, L. y Espinasa, R. (2015). Oil sector performance and institutions. The case of Latin America. Washington: BID.

Banco Interamericano de Desarrollo (BID) (2018). Participación privada en infraestructura. Su evolución en Colombia y el apoyo del grupo BID. s. 1.: BID y BID Invest.
Bhaskar, R. (1998). The logic of scientific discovery. En Critical Realism. Essential Readings (pp. 48-103). London, New York: Routledge.

Beach, D. y Pedersen, R. B. (2013). Process-Tracing methods. Foundations and guidelines. United States of America: University of Michigan Press.

Befani, B. y Mayne, J. (2014). Process tracing and contribution analysis: A combined approach to generative causal inference for impact evaluation. IDS Bulletin, 45(6), 17-36. 10.1111/1759-5436.12110

Bennett, A. (2010). Process tracing and causal inference. En Rethinking Social Inquiry. Diverse Tools, Shared Standards (pp. 207-219). Lanham: Rowman \& Littlefield Publishers.

Bobrow, D. (2006). Policy design: Ubiquitous, necessary and difficult. Handbook of Public Policy (pp. 7596). London: SAGE Publications.

Bonet, J. y Urrego, J. (2014). El Sistema General de Regalías: ¿̨mejoró, empeoró o quedó igual? Documentos de trabajo sobre economía regional, (198), 1-44.

Boole, G. (1854). An Investigation of the Laws of Thought on which are Funded the Mathematical Theories of Logic and Probabilities. London y Cambridge: Walton and Maberly; Macmillan and Co.

Borja, R. (2012). Enciclopedia de la politica (tomos I y II). México: Fondo de Cultura Económica.

Brinks, D., Levitsky, S. y Murillo, M. V. (2019). Understanding Institutional Weakness. Power and Design in Latin American Institutions. Cambridge: Cambridge University Press. 10.1017/9781108772211.

British Petroleum (BP) (2008). Statistical review of world energy. London: BP.

British Petroleum (BP) (2018). Statistical review of world energy. London: BP.

Capano, G. y Howlett, M. (2019). Causal logics and mechanisms in policy design: How and why adopting a mechanistic perspective can improve 
policy design. Public Policy and Administration, 1-22. 10.1177/0952076719827068

Collier, D. (2011). Understanding Process Tracing. PS: Political Science \&Amp; Politics, 44(4), 823-830. 10.1017/S1049096511001429

Cowater Sogema (2018). Validación de Colombia. Informe final de Validación. Recuperado de https://eiti. org/sites/default/files/documents/es_final_validation_report_country_columbia.pdf.

Departamento Nacional de Planeación (DNP) (2003). Plan Nacional de Desarrollo 2002-2006: Hacia un Estado comunitario. Bogotá: Imprenta Nacional de Colombia.

Departamento Nacional de Planeación (DNP) (s. f.). Cifras de inversión en infraestructura 1987-2011. Recuperado de https://www.dnp.gov.co/programas/participaci\%C3\%B3n-privada-\%20y-enproyectos-de-infraestructura/Paginas/inversionprivada.aspx.

Ecopetrol (2009). Reporte de sostenibilidad 2009. Bogotá: Ecopetrol.

Ecopetrol (2012). Reporte integrado de gestión sostenible 2011. Bogotá: Ecopetrol.

Ecopetrol (2014). Reporte integrado de gestión sostenible 2013. Bogotá: Ecopetrol.

Ecopetrol (2016). Reporte integrado de gestión sostenible 2015. Bogotá: Ecopetrol S.A.

Iniciativa de Transparencia de las Industrias Extractivas (етті) (2019). El estándar EITI. El estándar global para la buena gobernanza del petróleo, gas y recursos minerales. Oslo: Secretariado Internacional eIтI.

Eslava, A. (2010). Análisis neoinstitucional de políticas públicas. En Enfoques para el análisis de politicas públicas (pp. 97-124). Bogotá: Universidad Nacional de Colombia.

Fairfield, T. y Charman, A. (2016). Formal Bayesian Process Tracing: Guidelines, Opportunities, and Caveats (documento de trabajo). London: The London School of Economics and Political Science.

Fontaine, G., Narváez, I. y Fuentes, J. L. (2018). Policy mixes against oil dependence: Resource nationalism, layering and contradictions in Ecuador's energy transition. Energy Research \& Social Science, 47, 56-68.

Fontaine, G., Medrano, C. y Narváez, I. (2019). The Politics of Public Accountability: Policy Design in Latin American Oil Exporting Countries. Quito: Palgrave MacMillan.

Fontaine, G. (2015). El análisis de politicas públicas. Quito: FLACso Ecuador.

Fox, J. (2007). The uncertain relationship between transparency and accountability. Development in Practice, 17(4-5), 663-671. 10.1080/09614520701469955

Ganuza, E., Paes de Barros, R. y Vos, R. (2001). Efectos de la liberalización sobre la pobreza y la desigualdad. En Liberalización, desigualdad y pobreza: América Latina y el Caribe en los 90 (pp. 77-116). Buenos Aires: Editorial Universitaria.

Hall, P. (2003). Aligning ontology and methodology in comparative research. En Comparative Historical Analysis in the Social Sciences (pp. 373-404). Cambridge: Cambridge University.

Hernández, J. (2018). La anticorrupción en Colombia, el agente encubierto y la función de inteligencia. Revista Prolegómenos Derechos y Valores, 21(41), 99-114. http://dx.doi.org/10.18359/prole.3332

Hood, C. (1986). The tools of Government. New Jersey: Chatham House Publishers.

Howlett, M., Ramesh, M. y Perl, A. (2009). Studying Public Policy. Policy Cycles and Policy Subsystems. Toronto, Oxford, New York: Oxford University Press.

Isunza Vera, E. y Gurza Lavalle, A. (2015). El control democrático del Estado en Latinoa- 
mérica. Desacatos, (49), 6-7. http://www. scielo.org.mx/scielo.php? pid=S 1607 050X2015000300006\&script=sci_arttext

Jackson, P. (2016). The conduct of inquiry in international relations. Philosophy of science and its implications for the study of world politics. New York: Routledge Taylor \& Francis Group.

Kuhn, T. (1971). La estructura de las revoluciones cientificas. México: Fondo de Cultura Económica.

Lascoumes, P. y Simard, L. (2011). L'action publique au prisme de ses instruments. Introduction. Revue française de science politique, 61(1), 5-22. 10.3917/rfsp.611.0005.

Lascoumes, P. y Le Gales, P. (2007). Introduction: Understanding public policy through its instruments. From the nature of instruments to the sociology of public policy instrumentation. Governance: An International Journal of Policy, Administration, and Institutions, 20(1), 1-21.

Lowndes, V. y Roberts, M. (2013). Why Institutions Matter. The New Institutionalism in Political Science. New York: Palgrave Macmillan.

March, J. y Olsen, J. (1989). Rediscovering Institutions. The Organizational Basis of Politics. New York: The Free Press.

March, J. y Olsen, J. (2010). Reglas e institucionalización de la acción. Organización e instituciones (pp. 115141). México: Siglo xxi Editores.

Martínez, L. (2016). Sources of Revenue and Government Performance: Evidence from Colombia (Documento de trabajo). London: London School of Economics and Political Science.

Mejía, A. (2013). The impact and effectiveness of accountability and transparency initiatives: The governance of natural resources. Development Policy Review, 31(S1), S89-S105.

Mesa, L., Rivera, M. y Romero, J. (2011). Descripción general de la Inferencia Bayesiana y sus aplica- ciones en los procesos de gestión. Laboratorio de modelamiento y simulación, (2), 1-28.

Michot, M., Farrell, M. y Volkov, D. (2007). Hydrocarbon sector organization and regulation. En Energy Cooperation in the Western Hemisphere: Benefits and Impediments (pp. 444-475). Washington DC: Center for Strategic and International Studies.

Natural Resource Governance Institute (2017). 2017 Resource Governance Index. New York: Natural Resource Governance Institute.

Observatorio de Transparencia y Anticorrupción. Indicador de avance en la implementación de la convención de Naciones Unidas contra la Corrupción (UNCAC) (s. f.). Recuperado de http:// www.anticorrupcion.gov.co/Paginas/indicadoravance-implementacion-convencion-nacionesunidas-contra-corrupcion.aspx

O’Donnell, G. (1997). Rendición de cuentas horizontal y nuevas poliarquías. Nueva Sociedad, (152), 143-167.

Paz, B. y Fontaine, G. (2018). A causal mechanism of policy innovation: The reform of Colombia's oil-rents management system. Revista de Estudios Sociales, (63), 2-19. https://dx.doi.org/10.7440/ res63.2018.01

Peruzzotti, E. y Smulovitz, C. (2002). Accountability social: la otra cara del control. En Controlando la politica. Ciudadanos y medios en las democracias latinoamericanas (pp. 1-39). Buenos Aires: Grupo Editorial Temas.

Peters, B. G. (2018). Policy Problems and Policy Design. Cheltenham: Edward Elgar Publishing.

Pierre, J. y Peters, B. G. (2000). Governance, Politics and the State. London: Palgrave Macmillan.

Ragin, Ch. (1987). The comparative method. Moving beyond qualitative and quantitative strategies. California, London: University of California Press. 
Ramírez, D., Narváez, I. y Fontaine, G. (2018). ¿Por qué persisten los déficits de control social?: la consulta previa, libre e informada en el marco de la política petrolera ecuatoriana. Ciencia Politica, 13(25), 21-49.

Relly, J. (2012). Examining a model of vertical accountability: A cross-national study of the influence of information access on the control of corruption. Government Information Quarterly, (29), 335-345.

Relly, J. y Sabharwal, M. (2009). Perceptions of transparency of government policymaking: A crossnational study. Government Information Quarterly, (26), 148-157.

Ríos, A., Cortés, A., Suárez, M. y Vélez, L. (2014). Accountability: aproximación conceptual desde la filosofía política y la ciencia política. Colombia Internacional, (82), 261-288.

Romero, A. y Fontaine, G. (2017). Globalización, gobernanza y diseño de políticas públicas. La transformación de los sistemas políticos y de los Estados. La visión y los retos en Europa y América Latina en el contexto de la globalización (pp. 59-73). Barcelona, Santa Fe: Anthropos Editorial y Universidad Nacional del Litoral.
Salamon, L. (2002). The new governance and the tools of public action: an introduction. En The tools of government: A guide to the new governance (pp. 1-47). New York: Oxford University Press.

Sayer, A. (2010). Method in Social Science. A Realist Approach. New York: Routledge Taylor \& Francis Group.

Schneider, C. y Rohlfing, I. (2013). Combining QCA and process tracing in set-theoretic multi-method research. Sociological Methods \& Research, 42(4), 559-597. 10.1177/0049124113481341

Smith, A. (1958). Investigación sobre la naturaleza y causas de la riqueza de las naciones. México: Fondo de Cultura Económica.

Suárez, Ó. (2010). Sobre la separación de poderes en Colombia. Derecho y Realidad, (16),145-158.

Transparencia Internacional (2017). Indice de Percepción de la Corrupción 2017. Recuperado de https:// www.transparency.org/news/feature/corruption_ perceptions_index_2017.

van Evera, S. (1997). Guide to Methods for Students of Political Science. Ithaca: Cornell University Press.

Walter, M. (2016). Extractives in Latin America and the Caribbean: The basics. Technical Note IDBTN-907, 1-20. 